\title{
Infective endocarditis in an infant complicated with ischaemic stroke and mycotic aneurysm of the aorta
}

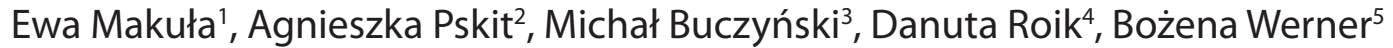 \\ 'Student Scientific Circle, Department of Paediatric Cardiology and General Paediatrics, Medical University of Warsaw, \\ Warsaw, Poland \\ 2Department of Paediatric Cardiology and General Paediatrics, Children's Clinical Hospital, Warsaw, Poland \\ ${ }^{3}$ Department of Cardiac and General Paediatric Surgery, Medical University of Warsaw, Warsaw, Poland \\ ${ }^{4}$ Department of Paediatric Radiology, Medical University of Warsaw, Warsaw, Poland \\ ${ }^{5}$ Department of Paediatric Cardiology and General Paediatrics, Medical University of Warsaw, Warsaw, Poland
}

\section{ABSTRACT}

Infective endocarditis is rare in children. Its incidence is higher among patients with congenital heart diseases. Neurological symptoms caused by emboli can be the first manifestation of the disease and can lead to diagnosis.

We present an infant after cardiosurgery for coarctation of the aorta, in whom unusual formation of mycotic aneurysm at the operated place occurred. The patient was admitted to the emergency department with feeding difficulties. Physical examination revealed left hemiparesis and a new heart murmur on the apex. Laboratory and imaging tests confirmed the diagnosis of infective endocarditis with a 6-mm vegetation on the mitral valve complicated with ischaemic stroke. A chest X-ray performed in the sixth week of treatment revealed an oval-shaped shadow in the area of the descending aorta. Computed tomography angiography confirmed a mycotic aneurysm formation in that location. The patient was operated on with good outcome.

\section{KEY WORDS:}

infective endocarditis, stroke, mycotic aneurysm, infant.

\section{INTRODUCTION}

Infective endocarditis (IE) is a rare disease in the paediatric population. It affects $0.04 \%$ of children per year [1]. The risk factors are congenital heart disease (CHD), cardiac surgery performed in the last six months, or age below three years [1-3]. Complication occurrence can be the first manifestation of the IE, which leads to diagnosis. There is no publication on a mycotic aneurysm formation at the place of corrected coarctation of the aorta $(\mathrm{CoA})$ in the course of IE.

\section{CASE REPORT}

We present a case of a 4.5-month-old boy with ventricular septal defect (VSD) after surgical repair of oesophageal atresia and cardiosurgical treatment of CoA with hypoplastic aortic arch. The boy was admitted to the emergency department with feeding difficulties and inconsolable crying.

The patient was born from first pregnancy by spontaneous vaginal delivery at 40 weeks of gestation, his birth weight was 3840 grams and Apgar score was 8-9-10

\section{ADDRESS FOR CORRESPONDENCE:}

Bożena Werner, Department of Paediatric Cardiology and General Paediatrics, Medical University

of Warsaw, 63A Żwirki i Wigury St., 02-091 Warsaw, Poland, ORCID: 0000-0002-6416-4106,

e-mail: bozena.werner@wum.edu.pl 
points. Congenital heart defects were diagnosed prenatally. The oesophageal atresia was recognised after the birth, and surgical treatment was performed on the first day of life. The surgical correction of CoA was postponed due to the raised inflammatory markers during the postoperative period. Intravenous infusion of prostaglandin E1, which was administered after birth, was continued to maintain arterial duct patent. CoA was operated on the $17^{\text {th }}$ day of life using an extended end-to-end method. The post-operative period was complicated by systemic infection caused by Enterococcus faecalis, treated according to antibiogram with vancomycin and amikacin until negative blood culture results were obtained. At the ninth week of age the child was discharged with good clinical and echocardiographic outcome of surgical treatment.

The physical examination after admission to the emergency department two months later revealed that the child was in mild condition, body temperature was normal, and no skin lesions were present apart from the scar after the left thoracotomy. Muscle tone was decreased on the left side of the body, and left extremity paresis and dropping of left corner of the mouth were observed. Retractions of lower intercostal spaces and clear breath sounds were present during the examination. His heart rate was 145 beats per minute, blood pressure was $105 / 80 \mathrm{~mm} \mathrm{Hg}$, and oxygen saturation was $99 \%$. The arm-leg systolic blood pressure gradient was about 10-15 mm Hg. Heart auscultation revealed systolic murmur 3-4/6 in the Levine scale in the Erb's point, radiating to the liver - a typical murmur for ventricular septal defect - and systolic murmur 2/6 in the Levine scale on the apex - the murmur of mitral regurgitation. The liver edge was palpable about $2 \mathrm{~cm}$ below the right costal margin, and the spleen $-1 \mathrm{~cm}$ below the left costal margin. Pulse was palpable symmetrically on femoral arteries.

Laboratory testing was performed. The C-reactive protein concentration level was elevated to $3.6 \mathrm{mg} / \mathrm{dl}$ (the norm was $0-1 \mathrm{mg} / \mathrm{dl}$ ), but procalcitonin, leucocytosis, parameters of liver, and kidney function were not elevated. Positive blood culture with growth of Enterococcus faecalis was observed. Chest X-ray showed cardiomegaly. Two-dimensional transthoracic echocardiography revealed a 6-mm vegetation on the anterior leaflet of the mitral valve, posterior leaflet thickening, and grade II mitral regurgitation (Fig. 1). IE was diagnosed. The patient was treated with ampicillin and streptomycin.

Magnetic resonance imaging (MRI) showed ischaemic stroke in the territory of the right middle cerebral artery, including the cortex, white matter, and deep brain structures. Dexamethasone, furosemide, and piracetam were administered. Due to seizure episodes and increased muscle tension, phenobarbital and mannitol were added to the treatment.

Neurological improvement was observed. Moreover, during the hospitalisation there was no fever and no symptoms of heart failure. Further echocar-

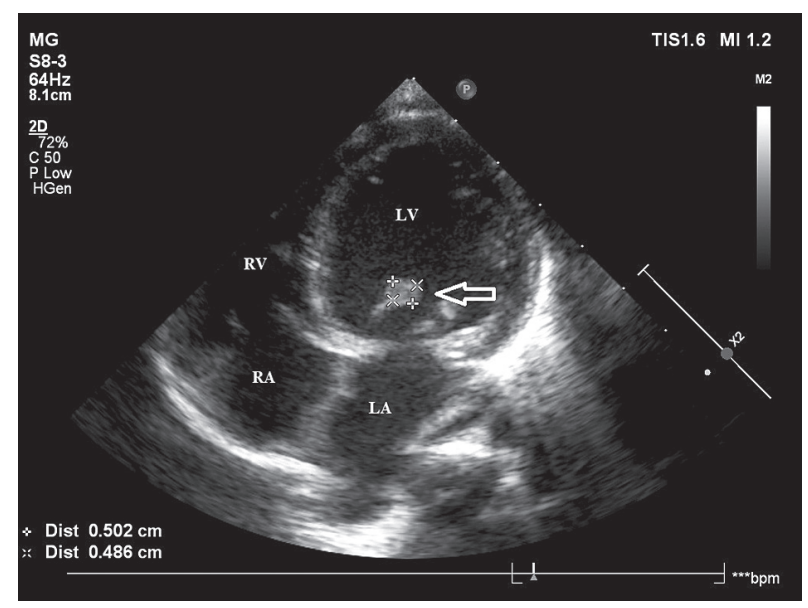

FIGURE 1. The echocardiographic trans-thoracic apical four-chamber view of the heart demonstrating the vegetation attached to the anterior leaflet of the mitral valve (arrow). LV - left ventricle, LA - left atrium, RV - right ventricle, RA - right atrium



FIGURE 2. The anteroposterior projection chest radiograph demonstrating an oval-shaped shadow in the area of descending aorta corresponding to the diagnosed aneurysm location (arrow)

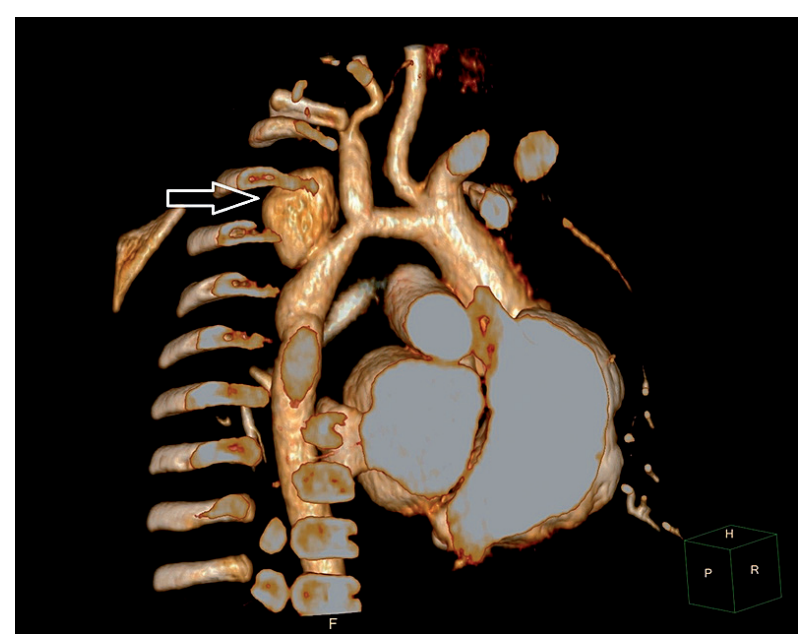

FIGURE 3. Angiography of computed tomography demonstrating saccular outpouching of the descending aorta below the origin of the left subclavian artery (arrow) 
diographic evaluation showed reduction in the size of the vegetation and trivial mitral regurgitation. At the end of the six-week treatment the chest X-ray revealed an oval-shaped shadow in the area of the descending aorta (Fig. 2). Computed tomography angiography (angioCT) showed an aneurysm-like enlargement of the aortic wall located $4.5 \mathrm{~mm}$ below the origin of the left subclavian artery. The diameter of this structure was $15 \mathrm{~mm} \times 17 \mathrm{~mm}$ with $7 \mathrm{~mm}$ ostium (Fig. 3). The neonate was qualified for aortic wall repair and VSD closure during a single-stage surgery. Transfontanellar ultrasound scan, brain MRI, and neurosurgical consultation were performed. No contraindications to surgery were stated.

Cardiac surgery was performed with extracorporeal circulation and deep hypothermic circulatory arrest. A 2-cm saccular aneurysm of the descending aorta surrounded by inflamed tissues was dissected. CorMatrix was used as a patch arterioplasty for the aorta. The hypoplastic aortic arch was widened and the VSD was closed. The histopathology report confirmed inflammatory aetiology of the aneurysm. The follow-up echocardiography and chest X-ray showed good outcome of the surgery.

\section{DISCUSSION}

IE is an inflammatory disease involving the endocardium and large vessels with the presence of infected material formation due to endovascular infection located inside the heart or vessels.

This paper presents a case of a four-month-old boy with VSD, who underwent surgical repair of oesophageal atresia and correction of CoA. An infant was diagnosed with IE complicated by ischaemic stroke and infectious (mycotic) aneurysm. CHD, cardiac surgery, age less than three years, or rheumatic heart disease are the main risk factors; however, data from the literature show that in the past two decades morbidity of rheumatic fever has become a rare underlying condition in developed countries [1, 4-6]. Interestingly, it has been shown that corrective heart surgery, especially with the use of prosthetic material, can be a greater risk factor than the presence of the unrepaired CHD itself [7]. Rushani et al. noted that the risk of IE development is more than five times higher in the first six months after cardiac surgery [1]. Notably vulnerable to IE are also children under the age of one year, because of the possibility of early CHD correction, particularly with extracorporeal circulation use and relevant postoperative care (including central catheter insertion) $[7,8]$.

In our case the IE was associated with enterococcal bacteraemia, which could be associated with oesophageal corrective surgery or long-term venous access [7, 9]. Nevertheless, the risk of enterococcal endocarditis development is much higher in community-acquired infections [9]. It was also observed to be a predominant aetiology in children $<2$ years of age [4]. The authors point out that the most common pathogens are streptococci and staphylococci, especially Staphylococcus aureus. In comparison with previous decades, the proportion of IE cases caused by coagulase-negative staphylococci, enterococci, Gram-negative bacilli, and yeast has increased as well as non-streptococcal and non-staphylococcal pathogens associated with hospital-acquired IE [8].

In our patient the IE occurred probably due to reactivation of the high-level gentamicin resistance (HLGR) Enterococcus faecalis infection. According to Ronen BenAmi et al., who postulated broadening of the time frame of hospital-acquired IE up to six months, and the same pathogen infection, we consider this case of IE to be nosocomial [10].

In comparison with the pre-antibiotic period, in the modern era the crude mortality rate has remained unchanged, but the overall complication rate is significantly lower. Complications, however, are more common in community-acquired endocarditis, mainly due to late diagnosis and treatment $[8,11]$.

In this case the complications were ischaemic stroke and infectious (mycotic) aneurysm formation. The complications of IE can be classified into cardiac and extracardiac sequelae. Cardiac complications include congestive heart failure, valvular dysfunction, sinus of Valsalva aneurysm rupture, and, less commonly, emboli to the coronary arteries. Extracardiac complications are, most importantly, the result of septic emboli $[3,5,6]$. Emboli usually occur within 10 days of the onset of symptoms, and the vast majority appear before the onset of therapy [12]. Among the proven risk factors of embolic episodes are mobile vegetations $>10 \mathrm{~mm}$ in size [12-14]. Emboli most often occur in the central nervous system, which was observed in the presented case. The second most frequent location is the spleen. Less often, emboli occur in skin, kidneys, coronary arteries, or lungs [12]. The consequences might include the development of neurological sequelae, such as ischaemic stroke, brain abscess, haemorrhage, or mycotic aneurysm formation in any systemic artery [5]. Two possible routes of arterial wall infection and infectious aneurysm formation are septic embolism at the site of vasa vasorum and bacteraemia, both resulting in degeneration of the arterial wall. Mycotic aneurysm formation in the setting of bacteraemia alone is rare and constitutes a rare clinical entity (the endothelial lining of the tunica intima is an effective barrier, and the high velocity of arterial flow makes the colonisation difficult), unless there is significant turbulence $[3,15]$. In the presented case turbulent blood flow was present at the site of previously corrected CoA.

AngioCT showed homogenous contrast-enhancing saccular aneurysm of the descending aorta. The inflammatory changes of surrounding tissues were seen intraoperatively, and inflammatory cell infiltration of the aortic wall was confirmed in histopathological examination. According to guidelines, angioCT is the imaging modality 
of choice for the evaluation of mycotic aneurysms. Cardiac magnetic resonance angiography has also become a suitable alternative to angioCT, particularly in the case of the presence of contraindications for the use of contrast media. Mycotic aneurysms are, characteristically, contrast-enhancing saccular outpouchings $[15,16]$. Other imaging findings suggestive of infectious aneurysms include soft-tissue inflammation around the vessel, presence of intramural air, and perivascular air or fluid collection [16].

In the presented case the aetiological factor was the HLGR strains with a high-level aminoglycoside resistance, except for streptomycin. Streptomycin and ampicillin were administrated followed by the improvement of clinical status, reduction in the size of the vegetation observed in echocardiographic examination, and the decrease of inflammatory marker levels. According to guidelines for the management of enterococcal IE, effective antimicrobial therapy requires prolonged administration up to 4-6 weeks with the combination of ampicillin or penicillin and aminoglycoside: gentamicin or streptomycin. In the case of resistant strains, the treatment should be modified $[3,17]$.

Our patient underwent resection of the aneurysm and surgical aortoplasty with CorMatrix placement. Particularly important for the management is aggressive surgical debridement of the infected tissue followed by vascular reconstruction. A literature review shows two main approaches for the surgical repair: extra-anatomic bypass (indicated for infrarenal aneurysms) and in situ graft placement (for suprarenal or thoracic aneurysms) $[15,16]$. Alternatives include antibiotic therapy alone as a conservative management and endovascular stenting. Because of the young age of our patient, the stent size, and relevant lack of possibility for stent expansion he did not receive endovascular treatment.

\section{CONCLUSIONS}

In conclusion, the presented case emphasises the importance of watchful observation of patients who have undergone cardiac surgery. The onset of the IE might be insidious, and complications such as ischaemic stroke can be the first sign of the disease.

Mycotic aneurysm formation at the place of corrected CoA is unusual, but this complication can occur in any artery in the course of IE.

\section{DISCLOSURE}

The authors declare no conflict of interest.

\section{REFERENCES}

1. Rushani D, Kaufman JS, Ionescu-Ittu R, et al. Infective Endocarditis in Children With Congenital Heart Disease Clinical Perspective: Cumulative Incidence and Predictors. Circulation 2013; 128: 1412-1419.
2. Sun LC, Lai CC, Wang CY, et al. Risk factors for infective endocarditis in children with congenital heart diseases - A nationwide population-based case control study. Int J Cardiol 2017; 248: 126-130.

3. Habib G, Lancellotti P, Antunes MJ, et al. 2015 ESC guidelines for the management of infective endocarditis: the task force for the management of infective endocarditis of the European Society of Cardiology (ESC) endorsed by: European Association for Cardio-Thoracic Surgery (EACTS), the European Association of Nuclear Medicine (EANM). Eur Heart J 2015; 36: 3075-3128.

4. Coward K, Tucker N, Darville T. Infective endocarditis in Arkansan children from 1990 through 2002. Pediatr Infect Dis J 2003; 22: 1048-1052.

5. Baltimore RS, Gewitz M, Baddour LM, et al. Infective endocarditis in childhood: 2015 update: a scientific statement from the American Heart Association. Circulation 2015; 132: 1487-1515.

6. Werner B, Wróblewska-Kałużewska M, Kucińska B, et al. Clinical and therapeutic considerations in children with infective endocarditis. Med Wieku Rozw 2007; 11: 159-165.

7. Kelchtermans J, Grossar L, Eyskens B, et al. Clinical Characteristics of Infective Endocarditis in Children. Pediatr Infect Dis J 2018; doi: 10.1097/INF.0000000000002212 [Epub ahead of print]

8. Rosenthal LB, Feja KN, Levasseur SM, et al. The changing epidemiology of pediatric endocarditis at a children's hospital over seven decades. Pediatr Cardiol 2010; 31: 813-820.

9. Fernandez-Guerrero ML, Herrero L, Bellver M, et al. Nosocomial enterococcal endocarditis: a serious hazard for hospitalized patients with enterococcal bacteraemia. J Intern Med 2002; 252: 510-515.

10. Ben-Ami R, Giladi M, Carmeli Y, et al. Hospital-acquired infective endocarditis: should the definition be broadened? Clin Infect Dis 2004; 38: 843-850.

11. Hill EE, Herijgers $\mathrm{P}$, Claus $\mathrm{P}$, et al. Clinical and echocardiographic risk factors for embolism and mortality in infective endocarditis. Eur J Clin Microbiol Infect Dis 2008; 27: 1159-1164.

12. Deprele C, Berthelot P, Lemetayer F, et al. Risk factors for systemic emboli in infective endocarditis. Clin Microbiol Infect 2004; 10 : 46-53.

13. Thuny F, Disalvo G, Belliard O, et al. Risk of embolism and death in infective endocarditis: prognostic value of echocardiography: a prospective multicenter study. Circulation 2005; 112: 69-75.

14. Tischler MD, Vaitkus PT. The ability of vegetation size on echocardiography to predict clinical complications: a meta-analysis. J Am Soc Echocardiogr 1997; 10: 562-568.

15. Deipolyi AR, Rho J, Khademhosseini A, et al. Diagnosis and management of mycotic aneurysms. Clin Imaging 2016; 40: 256-262.

16. Spelman D. Overview of infected (mycotic) arterial aneurysm. Up to Date 2016; 22: 6-150.

17. Nigo M, Munita JM, Arias CA, et al. What's new in the treatment of enterococcal endocarditis? Curr Infect Dis Rep 2014; 16: 431. 